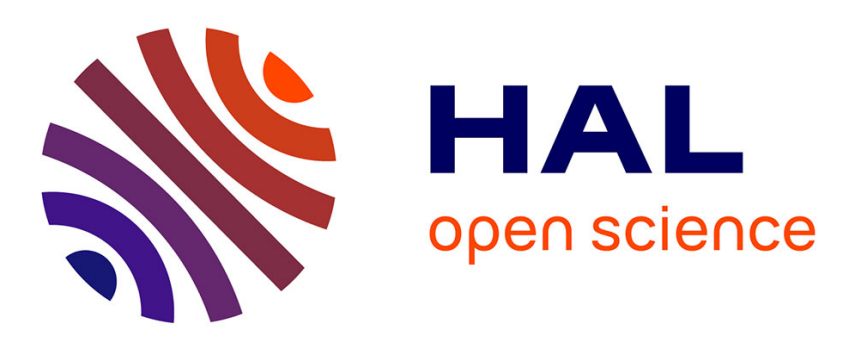

\title{
Fractal properties and characterization of road profiles
}

Pierrick Legrand, Jacques Lévy Véhel, Do Minh Tan

\section{To cite this version:}

Pierrick Legrand, Jacques Lévy Véhel, Do Minh Tan. Fractal properties and characterization of road profiles. FRACTAL04, Complexity and Fractals in Nature, 8th International Multidisciplinary Conference, Apr 2004, Vancouver, Canada. pp.189-198. inria-00576467

\section{HAL Id: inria-00576467 \\ https://hal.inria.fr/inria-00576467}

Submitted on 15 Mar 2011

HAL is a multi-disciplinary open access archive for the deposit and dissemination of scientific research documents, whether they are published or not. The documents may come from teaching and research institutions in France or abroad, or from public or private research centers.
L'archive ouverte pluridisciplinaire $\mathbf{H A L}$, est destinée au dépôt et à la diffusion de documents scientifiques de niveau recherche, publiés ou non, émanant des établissements d'enseignement et de recherche français ou étrangers, des laboratoires publics ou privés. 


\title{
FRACTAL PROPERTIES AND CHARACTERIZATION OF ROAD PROFILES
}

\author{
PIERRICK LEGRAND \\ IRCCyN, 1 rue de la Nöé, 44321 Nantes, France \\ e-mail: pierrick.legrand@irccyn.ec-nantes.fr \\ JACQUES LÉVY VÉHEL \\ IRCCyN, 1 rue de la Nöé, 44321 Nantes, France and \\ Projet Fractales, INRIA Rocquencourt, 78153 Le Chesnay Cedex, France \\ e-mail:vehel@irccyn.ec-nantes.fr \\ MINH-TAN DO \\ $L C P C$, route de Bouaye, 44340 Bouguenais, France
}

\begin{abstract}
A major problem in road engineering is to understand the mechanisms of friction between rubber and the road. Several authors have claimed that road profiles are fractal, and that this fractality is related to the friction properties of the road. We study road profiles obtained using tactile and laser captors. These profiles belong to different category characterized by different friction coefficients. We find that all our profiles indeed display strong fractal behaviour in terms of both correlation exponents and regularization dimension over a large range of scales. However, neither of these fractal parameters seem to be related to friction. We then use a local fractal parameter, namely the pointwise Hölder exponent. We show that this exponent does discriminate profiles which have different friction properties.
\end{abstract}

\section{Introduction and background}

An important problem in road engineering is to understand the mechanisms of friction between rubber and the road. This is a difficult problem, since friction depends on many parameters: The type of rubber, the type of road, the speed, ....

Several authors have shown that most road profiles are fractal ${ }^{1,2,9}$ on given ranges of scales. Such a property has obvious consequences on friction, some of which have been investigated for instance in ${ }^{1,4}$. The main idea is that, in the presence of fractal roads, all scales of irregularity contribute to friction ${ }^{3}$.

In this work, we verify that road profiles finely sampled using tactile and laser captors are indeed fractals. More precisely, we show that they have well-defined correlation exponent and regularization dimension over a wide range of scales. However, although we deal with various classes of profiles which have different friction coefficients, we find that such global fractal parameters are not able to discriminate between the profiles. This means that friction may have relatively low correlation with fractional dimensions or correlation exponents. We then compute a local parameter called the pointwise Hölder exponent. Our experiments show that this exponent allows to separate road profiles which have different friction coefficients.

\section{The road profiles}

Our profiles are provided by the LCPC (Laboratoire Central des Ponts et Chaussées). These profiles correspond to coatings with various gravel, and are 
characterized by different friction coefficients. A major aim of our study is to be able to relate these friction coefficients to fractal features of the profiles.

The samples consists of rectangular plaques with size $100 \times 150 \mathrm{~mm}^{2}$. Their surface is made of gravels cast into a a synthetic resin mould. The gravels come from thirteen different gravel pits, with a size varying between 6 and $10 \mathrm{~mm}$.

The manufacturing of the plaques consists in arranging the gravel in a flatbottomed rectangular mould, then filling the mould with fine sand and after that adding a quick setting resin. After removing from the mould, the plaques go through laboratory polishing cycles, that we describe briefly. A mixture of water and fine abrasives are thrown up to the surfaces with a $10 \mathrm{MPa}$ pressure. This processing induces certain changes in the microtexture of the gravel: The gravel originating from little polishable rocks keeps its initial microtexture; the one coming from highly polishable rocks loses its initial microtexture and becomes very smooth. Laboratory polishing thus makes it possible to emphasize the difference in microtexture between the different samples.

1D profiles have been sampled on the plaques through three different procedures: one using a tactile captor, and two using a laser captor. We briefly discuss these. Tactile measures

The details of the tactile sensor are as follows. The radius of the contact tip is 2 microns. The sensor's depth of field is $6 \mathrm{~mm}$. To avoid a potential locking of the contacting tip during its shifting on the tested surfaces, the gap in between the stones is filled with resin. Fifteen profiles are measured on each plaque within a zone of size approximately $75 \mathrm{x} 125 \mathrm{~mm}$. The length of the profiles varies from 12 to $25 \mathrm{~mm}$ according to the nature of the surfaces to be measured, reaching a total length of about $300 \mathrm{~mm}$ altogether. The sampling step is 4 microns, and the samples contains approximately 3100 to 6000 points.

Laser captor

The laser acquisition system developed at LCPC, based on an Imagine Optics captor, allows to modes: One uses a locking of the height, as the other does not. These two modes will be referred to in the following as locked and non-locked (see details in ${ }^{3}$ ). Again, fifteen profiles are measured on each plaque within a zone of size approximately $75 \times 125 \mathrm{~mm}$. The sampling step is 10 microns. The length of each profile is $125 \mathrm{~mm}$, resulting in a sample size of 12501 points.

In this paper, we shall focus on results pertaining to the analysis of a restricted number of profiles (results on other profiles are comparable):

- 3 tactiles profiles, with code names $B O U$ (friction coefficient 0.48), LRA (friction coefficient 0.63 ) and GRA (friction coefficient 0.775).

- 2 locked laser profiles, BOU again and another profile denoted CLE (friction coefficient 0.55 ).

- 3 non-locked laser profiles, $B O U, C L E$ and $Q B$ (friction coefficient 0.65).

As is apparent, the profiles in each acquisition procedure have clearly different friction properties. The friction for tactiles samples ranges from 0.48 to 0.775 , while for laser, it ranges between 0.48 and 0.65 . Besides checking the fractal behaviour of the profiles, our main aim is to investigate whether fractal parameters are able to 
discriminate between profiles with different friction coefficients. As an illustration, we show on figure 1 typical tactile profiles in the classes $B O U, L R A$ and $G R A$. Figure 2 displays typical $B O U$ and $C L E$ profiles in the locked laser mode
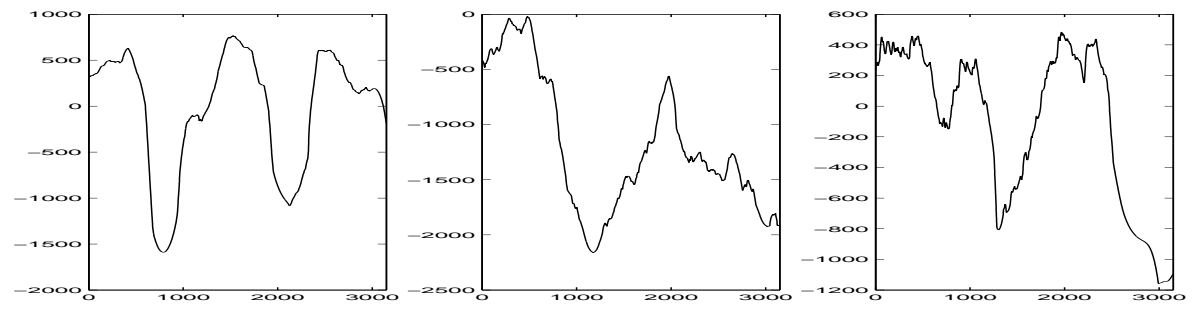

Figure 1. Typical BOU, LRA and GRA tactile profiles.
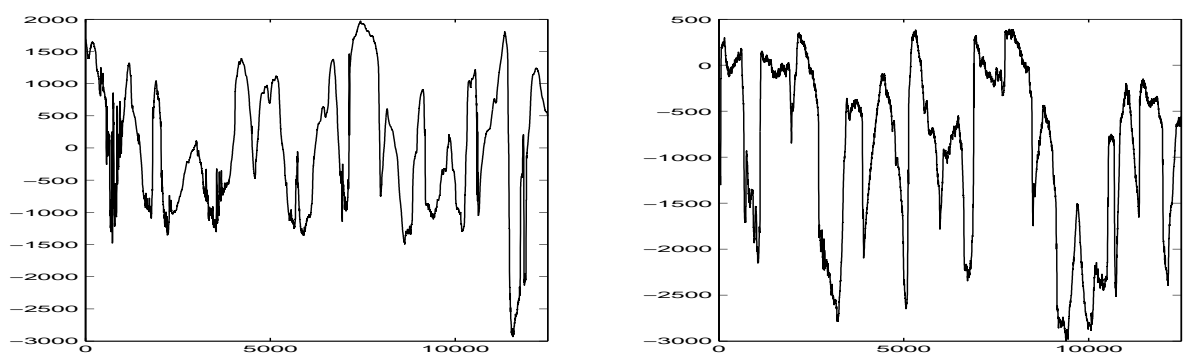

Figure 2. Typical $B O U$ and $C L E$ laser profiles.

\section{Fractal analysis}

We present briefly in this section the tools that we shall use to perform a fractal analysis of the profiles.

\subsection{Continuous wavelet transform (CWT)}

Recall that a wavelet is simply a function $\psi \in L^{2}(\mathbb{R})$ such that $\int_{\mathbb{R}} \psi(t) d t=0$. Usually, one requires in addition that $\psi$ be well localized in time and frequency, and has enough vanishing moments (i.e. $\int_{\mathbb{R}} x^{i} \psi(x) d x=0$ for $i=1 \ldots n$ ).

Définition 3.1.1. The continuous wavelet transform ${ }^{5}$ of a function $f \in L^{2}(\mathbb{R})$ is:

$$
C W T(a, b)=\frac{1}{\sqrt{a}} \int_{-\infty}^{\infty} f(t) \psi\left(\frac{t-b}{a}\right) d t
$$

As is well known, many fractal properties are related with the evolution of the wavelet coefficients $C W T(a, b)$ across scale, i.e. with respect to $a$. 


\subsection{Correlation exponent}

A classical fractal parameter we shall deal with is the correlation exponent. This exponent measures the speed of decay of the autocorrelation of a signal ${ }^{6}$. More precisely, assuming that $X(t)$ is stationary, denote $C(l)=E(X(t) X(t+l))$.

The signal $X$ has a well defined correlation exponent if $C(l) \sim l^{-\beta}$ with $\beta>0$ holds across a range of values of $l$. A particular case is of special interest: The signal $X$ is said to be long range dependant (LRD) if $C(l) \sim l^{-\beta}$ when $l$ tends to infinity, with $\beta>1$. The definition of LRD corresponds to the situation where the series $\sum_{l \in \mathbb{Z}} C(l)$ diverges.

\subsection{Regularization dimension (DimR)}

Fractional dimensions are one of the best known parts of fractal analysis. In this work, we shall deal with the so-called regularization dimension ${ }^{7}$. A heuristic explanation of DimR is the following. Start with a compactly supported signal $X$. For a given positive $s$, consider the convolution of $X$ with a Gaussian kernel of variance s. Let us denote by $X_{s}$ this regularized signal. Assume that $X$ is so irregular that it has infinite length. Since $X_{s}$ is $C^{\infty}$ for any positive $s$, it has finite length $L_{s}$. Furthermore, $X_{s}$ tends to $X$ when s tends to zero. The regularization dimension measures the speed of convergence of $L_{s}$ to infinity when $s$ tends to 0 (see figure 3 for an illustration on a road profile).

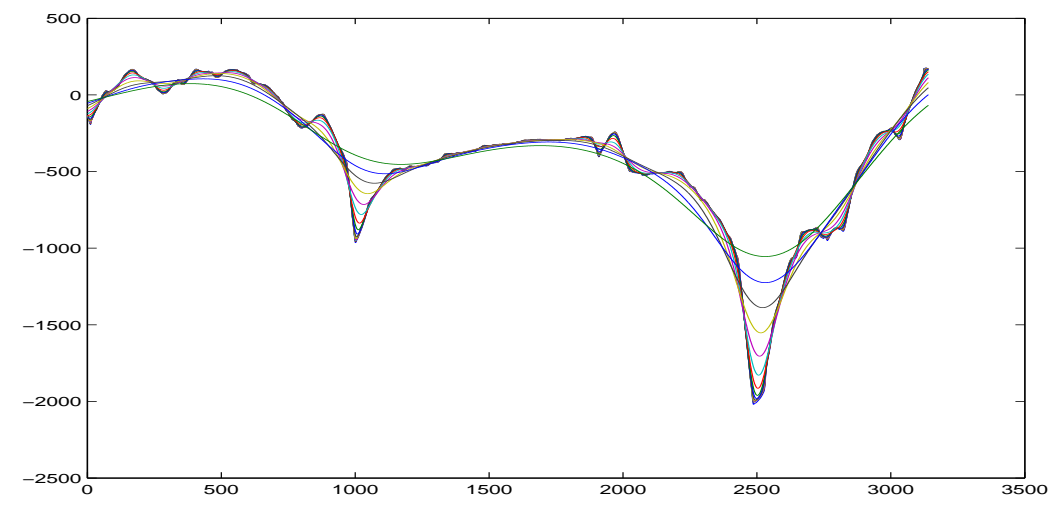

Figure 3. Computation of a regularization dimension on a profile through successive convolutions.

Let us now give a formal definition. Let $\Gamma$ be the graph of a bounded and compactly supported function $f: K \subset \mathbb{R} \rightarrow \mathbb{R}$. Let $\chi(t)$ be a kernel in the Schwartz class, and set, for $a>0, \chi_{a}(t)=\frac{1}{a} \chi\left(\frac{t}{a}\right)$. Let $f_{a}=f * \chi_{a}$. This function is infinitely smooth, and the length of its graph $\Gamma_{a}$ on $K$ is given by

$$
L_{a}=\int_{K} \sqrt{1+\left(f_{a}^{\prime}(t)\right)^{2} d t}
$$


Définition 3.3.1. The regularization dimension of (the graph of) $f$ is: $\operatorname{Dim} R(\Gamma)=$ $1+\lim \sup _{a \rightarrow 0} \frac{\log \left(L_{a}\right)}{-\log (a)}$

The regularization dimension coincides in many cases with the better known box dimension: This is in particular the case for all "classical" fractal signals such as Weierstrass functions, fractional Brownian motions, .... One may prove that the relation $\operatorname{Dim} R \leq \operatorname{Dim} B$, where $\operatorname{Dim} B$ is the box dimension, holds for any continuous function. This indicates that DimR is "finer" than the box dimension. The main advantage of the regularization dimension is that it leads to much more accurate estimations on sampled data than the box dimension. This is mainly because the number of smoothed versions is not restricted. Another reason is that DimR is less sensitive to noise than DimB. The interested reader may consult ${ }^{7}$ for more details.

From a practical point of view, a signal will be considered fractal if a plot of $\log \left(L_{a}\right)$ versus $\log (a)$ is linear in a certain range of values of $a$.

\subsection{Pointwise Hölder exponent}

In contrast with the correlation exponent and DimR, which are global quantities, the pointwise Hölder exponent $\alpha$ measures a local behaviour ${ }^{8}$. Its definition reads: Définition 3.4.1. Let $x_{0} \in \mathbb{R}$, and $s$ be a real number with $s>-1$. A function $f: \mathbb{R} \rightarrow \mathbb{R}$ belongs to $C_{x_{0}}^{s}$ if and only if there exist a constant $C$ and a polynomial $P$ of degree at most $[s]$ such that

$$
\left|f(x)-P\left(x-x_{0}\right)\right| \leq C\left|x-x_{0}\right|^{s} .
$$

The pointwise Hölder exponent of $f$ at $x_{0}$, denoted by $\alpha_{f}\left(x_{0}\right)$ or simply $\alpha$, is defined to be $\sup \left\{s: f \in C_{x_{0}}^{s}\right\}$.

When $0<\alpha<1$, it is given by the simple formula:

$$
\alpha=\liminf _{h \rightarrow 0} \frac{\log \left|f\left(x_{0}+h\right)-f\left(x_{0}\right)\right|}{\log |h|}
$$

Since $\alpha$ is defined at each point, one may associate to $f$ its Hölder function:

Définition 3.4.2. Let $f$ be a bounded function. The Hölder function of $f$ is the function which associates, to each $x, \alpha_{f}(x)$.

While the Hölder exponents and the Hölder function cannot tell whether a signal is "fractal", they provide a rich description of the local singularity structure of a signal. A small $\alpha_{f}(x)$ means that $f$ is irregular at $x$, and vice versa. For instance, if $f$ is $C^{1}$ at $x$, then $\alpha_{f}(x) \geq 1$; If $\alpha_{f}(x)<0$, then $f$ is discontinuous at $x$.

\section{Results}

We have computed the parameters described in the previous section on our road profiles. All the programs we have used are available in the software toolbox called FracLab. FracLab may be downloaded at www.irccyn.ec-nantes.fr/hebergement/ FracLab/ and http://fractales.inria.fr.

In the next subsection, we verify that the profiles display a fractal behaviour. Then, we use this property to characterize the signals. 


\subsection{Fractal behaviour}

\section{Energy and CWT}

One possible way to check for a fractal behaviour is to investigate the evolution of the energy in the signal with respect to scale. More precisely, let $E_{a}^{2}=\int[C W T(a, b)]^{2} d b$ denote the energy at scale $a$. A relation of the type $E_{a}^{2} \sim a^{\gamma}$ for some $\gamma$ and $a$ in a given range indicates that the energy decays as a power law in scale.

Figure 4 shows that such a relation is approximatively verified for most tactiles profiles across a large range of scales. Results on the other types of profiles are similar.
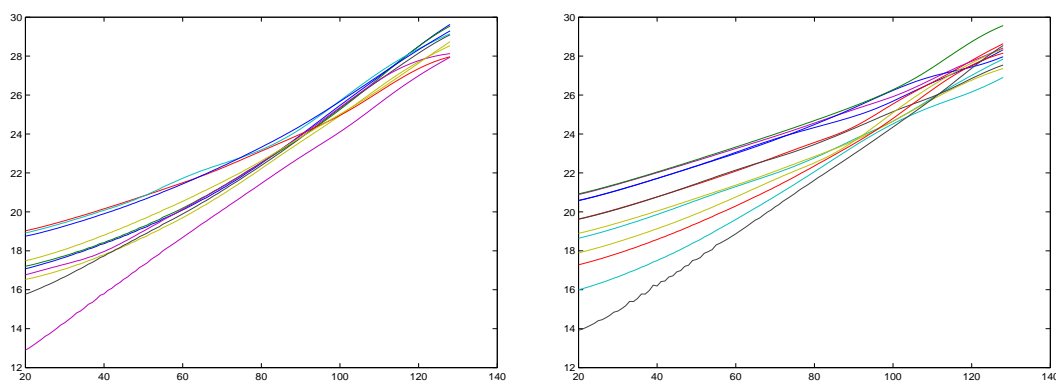

Figure 4. log-energy with respect to scale for the tactiles profiles BOU and LRA.

\section{Correlation exponent}

The results for the correlation exponent confirm the ones above. Figure 5 shows that, for tactiles profiles, the logarithm of the lag $l$ correlation $C(l)$ behaves linearly as a function of $\log (l)$ on almost all the range of possible values of $l$. Again, the same type of graphs are obtained with other profiles.
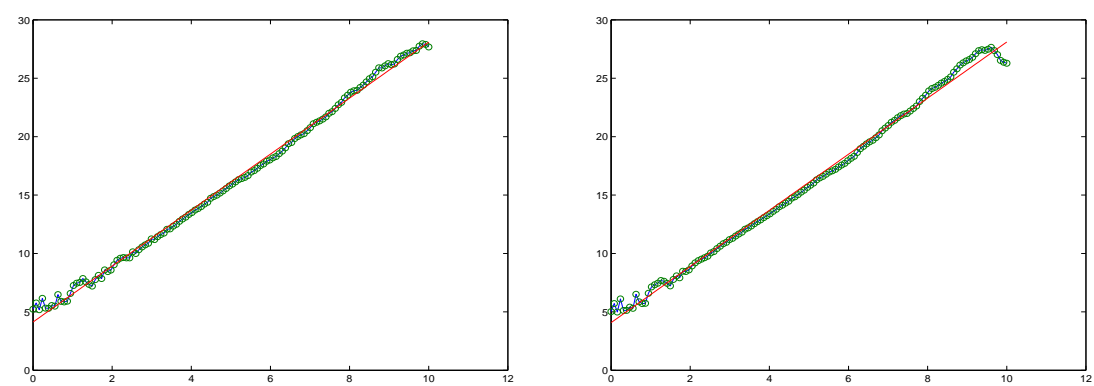

Figure 5. log-correlation as a function of the logarithm of the lag for the tactile profiles $L R A$ (left) and $G R A$ (right).

From a numerical point of view, the values of the exponents measured on various profiles range between 0.8 and 1.4. Thus, although a clear fractal behaviour is 
verified by all profiles, only some of them display LRD.

\section{Regularization dimension}

Figure 6 displays a typical behaviour of DimR on profiles. As one can see, the graph $\log \left(L_{a}\right)$ versus $\log (a)$ is not linear. There are however two linear regimes, one corresponding to a low regularization (i.e. high frequencies), and the other one valid for large smoothing, i.e. low frequencies. From the DimR point of view, it thus seems that our road profiles have two well-defined dimensions, indicating that the micro- and macro-textures behave in a different way.
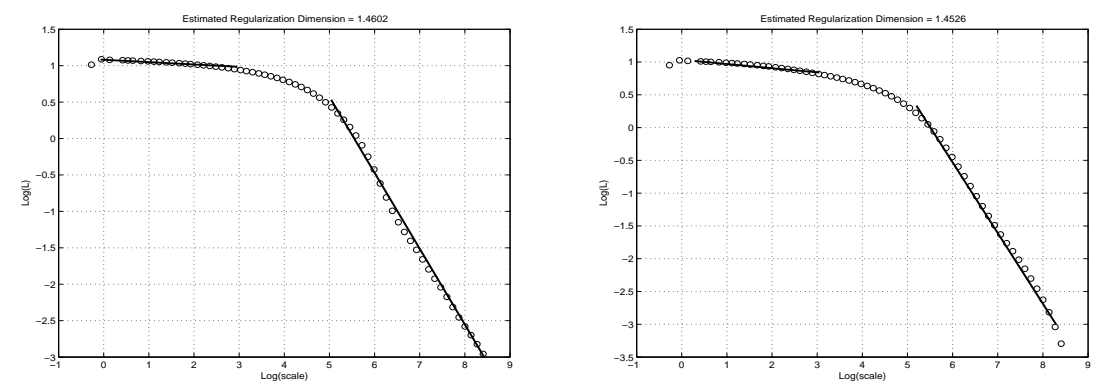

Figure 6. Evolution of the logarithm of the length of smoothed version of the tactile profiles $B O U$ (left) and $L R A$ (right) as a function of the logarithm of the smoothing parameter.

We note that the regularization dimensions range between 1.1 and 1.6 on our profiles. This indicates that the profiles vary between almost smooth and somewhat irregular.

\subsection{Profile characterization}

Although the profiles all clearly manifest a fractal behaviour, we have found that neither the correlation exponent nor the regularization dimension were able to characterize a given class of profiles. As a consequence, these parameters may not be used to explain the differences in friction of the various profiles. This is seen in a qualitative way on figure 7 . The correlation exponents are represented for all tactiles profile in the class $B O U$. Though all profiles show an excellent linear behaviour, the slopes of the 15 different samples vary a lot. Thus there is no single exponent that may be meaningfully attributed to a given class. Moreover, a quantitative analysis shows that the ranges of exponents for the different classes overlap a lot. It is thus not possible to separate the classes based on the information brought by the correlation exponent. The same comments apply to the regularization dimension.

This leads us to the following conclusion: If the fractality of the profiles is of any relevance for friction, this should be sought in local features rather than in global ones. Such a claim is supported by the fact that friction is mainly a local phenomenon. As a consequence, global measures of irregularity such as DimR or correlation exponents may be largely unrelated to the friction coefficient. In contrast, local regularity measures such as Hölder exponents should be strongly correlated with friction. We now proceed to investigate such correlations. 


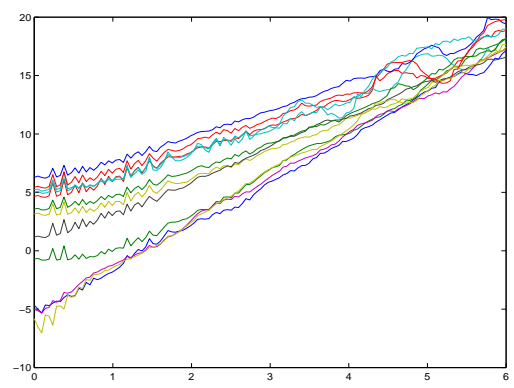

Figure 7. Correlation analysis of the $15 B O U$ tactiles profiles.

\section{Hölder function}

As an illustration, the Hölder functions for samples of the tactile profiles $B O U$ and $L R A$ are displayed on figure 8. Hölder functions for other profiles look similar.
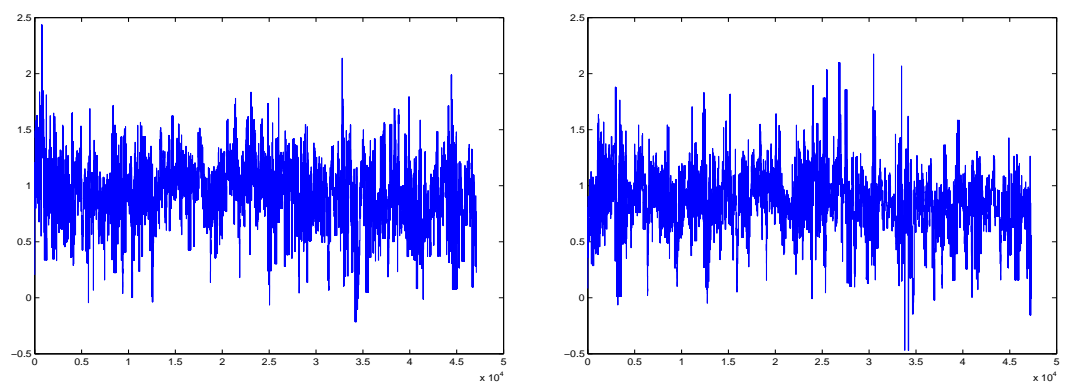

Figure 8. Hölder functions for the tactile profiles $B O U$ and $L R A$.

The Hölder function yields too rich an information for our purposes. We start by investigating the use of its median for profile characterization. Note that, while the median will subsume information pertaining to the whole signal, it is still a local parameter. It is thus radically different from a global parameter such as DimR. The use of the median (or the mean) of the Hölder function is consistent with the fact that the friction results from an average of many local interactions.

\section{Combination of DimR and the Hölder median}

We first compute the Hölder functions of all the profiles and all the samples. We then extract their median. Figure 9 shows an attempt to classify the different classes based on this median plus the regularization dimension. While this procedure works well for the two laser profiles in locked mode, it fails to separate the three classes of laser profiles in unlocked mode .

We now discuss another technique that makes a fuller use of the information brought by the Hölder function.

\section{Histograms of Hölder functions}

RoadProfils: submitted to World Scientific on July 12, 2003 

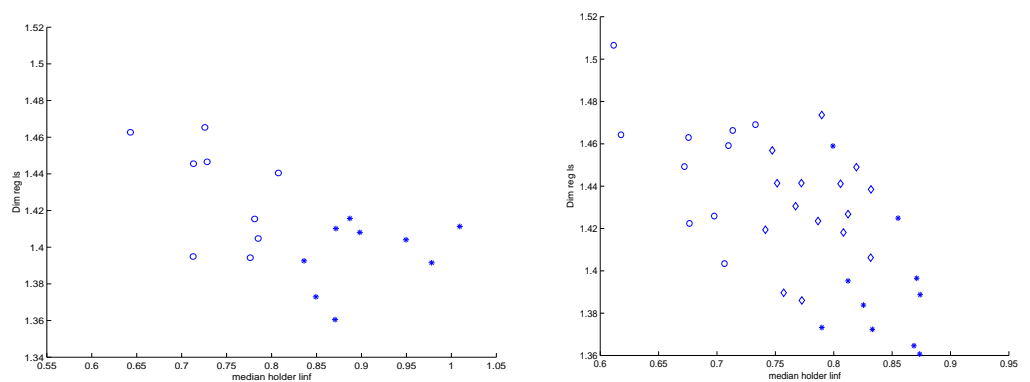

Figure 9. Classification of the profiles classes using the Hölder median (abscissa) and DimR (ordinates). The left plot displays locked mode laser profiles $C L E$ (circles) and $B O U$ (stars). The right plot shows unlocked mode laser profiles $C L E$ (circles), $Q B$ (diamonds) and $B O U$ (stars).

Instead of restricting to the median, we now study the histograms of the Hölder functions. More precisely, for each profile $P$, we compute ten histograms $H_{P}(\alpha)$ of the Hölder functions evaluated for ten "test" samples randomly chosen among the fifteen samples in profile $P$. We do this for the two locked mode laser profiles $B O U$ and $C L E$, and for the three unlocked mode laser profiles $B O U, C L E$ and $Q B$. See figure 10 .

The second step is to model these histograms as Gaussian processes. In other words, for each given profile and for each value of $\alpha$, we compute the mean $M_{P}\left(H_{P}(\alpha)\right)$ and variance $V_{p}\left(H_{P}(\alpha)\right)$ of $H_{P}(\alpha)$ evaluated over the ten test samples in this profile. Let $G_{P}\left(H_{P}(\alpha)\right)=\mathcal{N}\left(M_{P}\left(H_{P}(\alpha)\right), V_{p}\left(H_{P}(\alpha)\right)\right)$ denote the Gaussian distribution obtained for the histogram of the profile $P$ at value of the exponent equal to $\alpha$. To check whether a new, unknown, sample belongs to profile $P$, one first computes the histogram $h(\alpha)$ of its Hölder function. If the sample belong to $P$, we expect that $G_{P}(h(\alpha))$ is "large". A quantity that measures how the sample is "close" to profile $P$ is thus:

$$
T_{P}(h)=\int_{\mathbb{R}} G_{P}(h(\alpha)) d \alpha
$$

Any unknown sample is then attributed to the profile $P$ which maximizes $T_{P}(h)$.

We found that this method was able to classify with $100 \%$ success the five remaining samples in all classes. Our conclusions are thus as follows:

- Road profiles indeed display fractal behaviours in terms of both correlation exponent and regularization dimension over a large range of scales.

- Global fractal measures as are the correlation exponent and regularization dimension do not allow to characterize profiles.

- The local regularity information brought by the Hölder exponent allows to classify the profiles through a simple statistical procedure.

Future work will focus on relating the structure of the Hölder function with the friction coefficient of the profiles. 

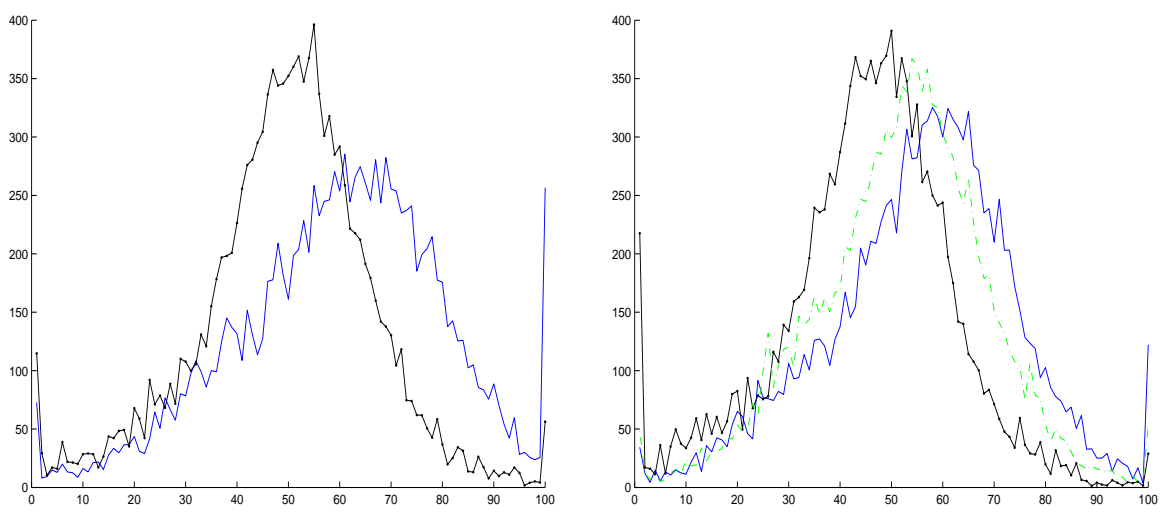

Figure 10. Left: Mean of the histograms of the Hölder functions for locked mode laser profiles $B O U$ (blue), $C L E$ (black). Right: Same, but for unlocked mode laser profiles $B O U$ (blue), $C L E$ (black) and $Q B$ (dotted green).

\section{References}

1. Z. RAdo, A Study of Road Texture and its Relationship to Friction, Phd Thesis, The Pennsylvania State University, 1994.

2. G. HeinRICH, Rubber friction on self-affine road tracks, Rubber Chemistry and Technology 70, 1997.

3. M.T. Do, H. Zahouani, Frottement pneumatique/chaussée, influence de la microtexture des surfaces de chaussée, JIFT, 2001.

4. M. KLÜPPEL, Rubber friction on self-affine road tracks, Rubber Chemistry and Technology 73, 578-606, 2000.

5. YY. Meyer, Ondelettes et Opérateurs, volume 1. Hermann, Paris, 1990.

6. J. Beran, Statistics for Long-Memory Processes, Chapman and Hall, New York, 1994.

7. F. RouefF, J. LeVy VÉHEL, A regularization approach to fractionnal dimension estimation, Fractal conference, 1998.

8. K. Daoudi, J. Lévy Véhel, Y. Meyer, Construction of Continuous Functions with Prescribed Local Regularity, Constructive Approximation. Vol. 14. Num. 3, pp.349-386, 1998.

9. M. Guglielmi, J. Levy-Vehel, Analysis and simulation of road profile by means of fractal model, Conference on Advances in Vehicle Control and Safety (AVCS 98) Amiens, 1998. 